\title{
Diazotrophic synchronous growth of a marine unicellular cyanobacterium, Synechococcus sp. strain Miami BG 043511, under aerobic and microaerobic/anaerobic conditions
}

\author{
Hisato Ikemotof and Akira Mitsuif
}

School of Marine and Atmospheric Science, University of Miami, 4600 Rickenbacker Causeway, Miami, Florida 33149, USA

\begin{abstract}
The growth attributes of an aerobic nitrogen-fixing Synechococcus strain, Miami BG 043511, under aerobic and microaerobid/anaerobic conditions were examined using conventional batch and synchronous culture methods. Generation times of this strain, estimated from the increase in cell density under aerobic and anaerobic batch culture conditions, were 19-23 $\mathrm{h}$ and 15-19 $\mathrm{h}$ at $30^{\circ} \mathrm{C}$, respectively. It seems, therefore, that atmospheric oxygen did not seriously affect diazotrophic growth in this strain. Under a periodic light-dark regime, cells grew synchronously even under microaerobic/anaerobic conditions. When the aerobic culture entered the light period, a peak of photosynthetic activity was followed by a peak of nitrogenase activity. In contrast, a peak of nitrogenase activity preceded a peak of photosynthetic activity under microaerobic/anaerobic conditions. In both cases, however, cell division was observed at or just after the peak of photosynthetic activity. The difference in the timing of the appearance of nitrogenase activity in microaerobiclanaerobic cultures was ascribed to the inability of cells to generate sufficient ATP under anaerobic dark conditions. Periodic changes in cellular carbohydrate content, associated with the periodic appearances of photosynthetic and nitrogen-fixing activities, were observed under both aerobic and microaerobic/anaerobic conditions. Cellular carbohydrate content increased from $10 \%$ to $60 \%$ of cell dry weight during the phase of photosynthesis under aerobic conditions, while it reached only $\mathbf{4 0} \%$ under microaerobic/anaerobic conditions. The amount of reserve polysaccharides required to support nitrogen fixation was larger in aerobic cultures than in microaerobidanaerobic cultures.
\end{abstract}

Keywords: Synechococcus sp., synchronous growth, microaerobic/anaerobic conditions, nitrogen fixation

\section{INTRODUCTION}

Despite the oxygen sensitivity of nitrogen fixation, several strains of unicellular cyanobacteria are capable of growing diazotrophically under aerobic photoautotrophic conditions (Fay, 1992; Gallon, 1992). Synechococcus strain Miami BG 043511 is one of these strains (Mitsui et al., 1983). In this strain, photosynthesis and nitrogen fixation occur concomitantly in conventional batch cultures, but

†Present address: Marine Biotechnology Institute, Japan.

$\ddagger$ Deceased.

Abbreviation: DCMU, 3-(3,4-dichlorophenyl)-1,1-dimethylurea. during different phases of the cell cycle under synchronously growing diazotrophic conditions (Mitsui et al., $1986,1987,1993)$. Since nitrogen fixation is known to be extremely sensitive to oxygen, it is of interest to compare the growth and the manner of the temporal separation of these incompatible reactions under aerobic and anaerobic conditions. However, we have not previously examined whether this strain is capable of growing synchronously under anaerobic nitrogen-fixing conditions. Our results now show that this strain can grow synchronously under both aerobic and microaerobic/anaerobic conditions, although the timing of the appearance of cell cycle events and the manner of carbohydrate accumulation and consumption are not the same. 


\section{METHODS}

Growth conditions. The marine unicellular cyanobacterium, Synechococcus sp. strain Miami BG 043511, was grown in a combined nitrogen-free medium as described previously (Leon et al., 1986). Cells were grown in a water-jacketed glass cylinder $(8.5 \mathrm{~cm}$ diameter, $3.3 \mathrm{l})$ at $30^{\circ} \mathrm{C}$ and $150 \mu \mathrm{mol}$ photons $\mathrm{m}^{-2} \mathrm{~s}^{-1}$. For aerobic and anaerobic cultures, approximately $3 \%$ carbon dioxide was mixed with air or with nitrogen gas, respectively. The gas mixture was introduced from the bottom of the culture vessels at the rate of $200-500 \mathrm{ml} \mathrm{min} \mathrm{m}^{-1}$ to bring the $\mathrm{pH}$ of the culture medium to $7 \cdot 6 \pm 0 \cdot 1$.

To obtain synchronized growth, cells at an early exponential phase were placed in artificial diurnal light-dark cycles (Mitsui et al., 1986; Mitsui \& Cao, 1988). Gassing was continued throughout the experiment in order to maintain $\mathrm{pH}$ and the level of dissolved oxygen in the medium.

Measurements of photosynthetic oxygen evolution and nitrogenase activities. The rate of photosynthetic oxygen evolution was measured at $30^{\circ} \mathrm{C}$ using a Clark-type oxygen electrode fitted to a water-jacketed reaction vessel $(1.8 \mathrm{ml})$ as described previously (Kumazawa \& Mitsui, 1985). Aerobically grown cells were transferred into the reaction vessel and bubbled with air enriched with carbon dioxide $(3 \%)$ for several minutes. In microaerobically/anaerobically grown cells, $\mathrm{NaHCO}_{3}(5 \mathrm{mM})$ and HEPES buffer $(20 \mathrm{mM}, \mathrm{pH} 7 \cdot 6)$ were added before placing cells into the reaction vessel. The suspension in the vessel was bubbled with argon gas for several minutes to remove any contaminating oxygen that was introduced during the sample handling. The rate of photosynthetic oxygen evolution was calculated from the net oxygen evolution after the light was turned on. Illumination was provided by a slide projector equipped with a $150 \mathrm{~W}$ tungsten lamp. Photon flux density, measured at the outer surface of the water jacket of the reaction vessel, was $2000 \mu \mathrm{mol}$ photons $\mathrm{m}^{-2} \mathrm{~s}^{-1}$. No photoinhibition or light limitation was observed with this light intensity throughout the experiment. Photosynthetic activity was also measured for the cells in the dark periods and shown as the potential photosynthetic activity at that time. Measurements were not carried out at constant intervals.

Nitrogenase activity was determined every 1 or $2 \mathrm{~h}$ as described previously (Leon et al., 1986) using the acetylene reduction method. For the assay of nitrogenase activity, the cell suspension $(5 \mathrm{ml})$ was placed into $25 \mathrm{ml}$ micro-Fernbach flasks, sealed with butyl rubber stoppers, and then $\mathrm{C}_{2} \mathrm{H}_{2}(2 \mathrm{ml})$ was injected into the flasks. The activity was measured under air for aerobic cultures; the gas phase of the flasks was replaced with argon prior to the injection of $\mathrm{C}_{2} \mathrm{H}_{2}$ for microaerobic/anaerobic cultures. Flasks were incubated in a water-bath shaker at $30^{\circ} \mathrm{C}$ for $30 \mathrm{~min}$. Illumination was provided from beneath by fluorescent tubes. Photon flux density at the bottom surface of the flasks was $150 \mu \mathrm{mol}$ photons $\mathrm{m}^{-2} \mathrm{~s}^{-1}$. For the dark assay, flasks were covered with aluminium foil. At the transition from dark to light as well as from light to dark, the activity was measured both in the dark and in the light.

Determination of cell population, dry weight and cellular carbohydrate content. Total cell numbers and those in the dividing stage were estimated using a Petroff-Hausser bacterial cell count chamber, after fixing $1 \mathrm{ml}$ samples with $50 \mu 10 \%$ Lugol solution.

Cell dry weight was measured after collecting the cells on preweighed glass micro-fibre filters (Whatman 934AH), washing twice with a small amount of distilled water, and drying at $90{ }^{\circ} \mathrm{C}$ for 24 h. Details were described previously (Leon et al., 1986).

The phenol/sulfuric acid method (Dubois et al., 1956) was used to measure the carbohydrate content of cell suspensions which had first been centrifuged (5000 $\mathrm{g}$ for $10 \mathrm{~min}$ ), washed once with fresh culture medium and resuspended in distilled water.

\section{RESULTS}

To compare the diazotrophic growth of strain Miami BG 043511 under aerobic and microaerobic/anaerobic conditions, cells were aerated with carbon dioxide-enriched air (aerobic) or carbon dioxide-enriched nitrogen gas (microaerobic/anaerobic). Since the photosynthetic oxygen production was not blocked under anaerobic sparging conditions, the culture condition was described as microaerobic/anaerobic. Under both conditions, cell numbers increased exponentially during the early stages of growth (Fig. 1). Aerobic growth was slightly slower than microaerobic/anaerobic growth, generation times estimated from the increase in cell density being 19-23 h and $15-19 \mathrm{~h}$, respectively. The generation time became longer at higher cell density.

When aerobically growing batch cultures were exposed to $12 \mathrm{~h}$ dark-12 h light cycles, synchronous cell division was observed following the first dark period (Fig. 2). When microaerobically/anaerobically growing cells were treated similarly, cell division also occurred in a segregated manner (Fig. 3). In both cases, more than $80 \%$ of the cell population were in the dividing stage of binary fission at the peak of cell division. However, there were distinct differences in the timing of cell cycle events between

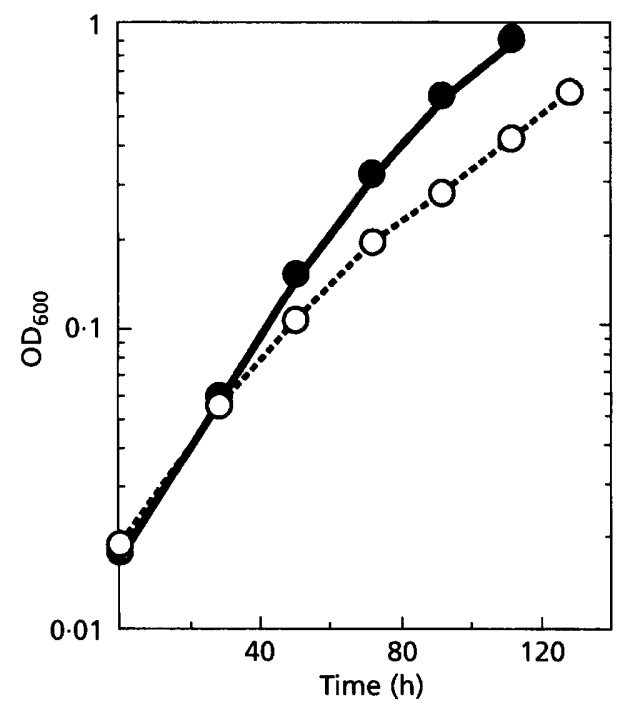

Fig. 1. Comparison of aerobic and microaerobic/anaerobic nitrogen-fixing growth of strain Miami BG 043511. Cultures were grown in continuous light and were sparged with either $3 \%$ carbon dioxide in air $(O)$ or $3 \%$ carbon dioxide in nitrogen (O). 


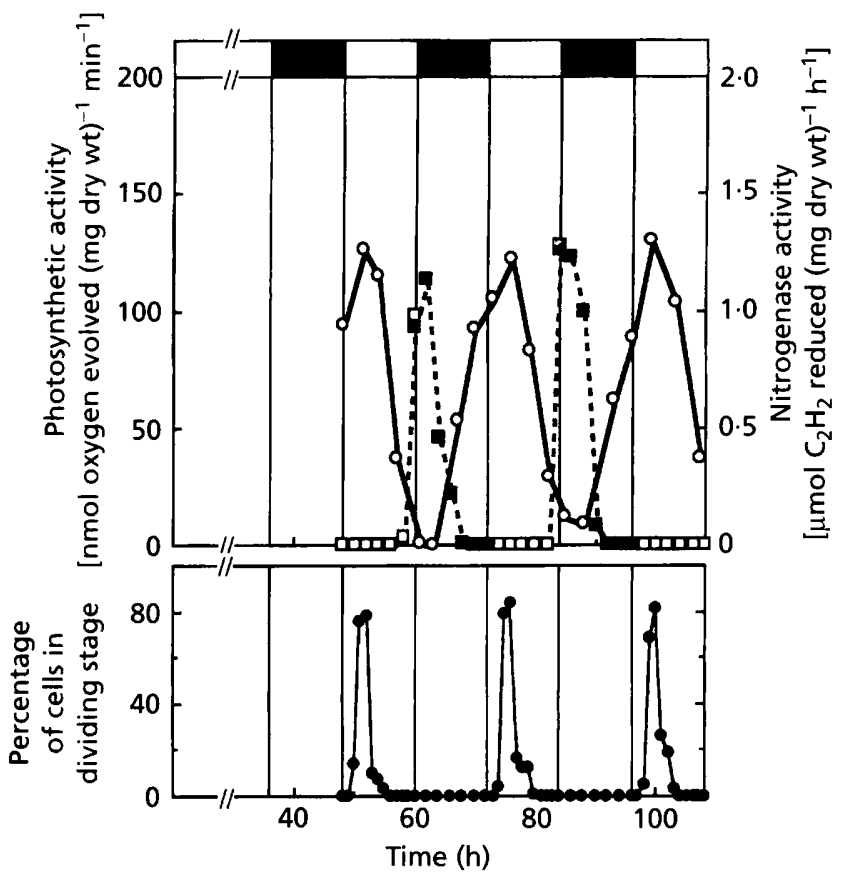

Fig. 2. Changes in nitrogen-fixing and photosynthetic activities of a culture placed in $12 \mathrm{~h}$ dark-12 h light cycles under aerobic conditions. Inoculation was carried out at $0 \mathrm{~h}$. Aeration (air/carbon dioxide) was continued throughout the experiment. Nitrogenase activity was measured under an atmosphere of air as the rate of $\mathrm{C}_{2} \mathrm{H}_{2}$ reduction either in the light $(\square)$ or in the dark ( $\square$ ), or in both the light and the dark at the light/dark and dark/light transitions. Photosynthetic activity $(O)$ was measured as the rate of net oxygen evolution. The activity was also measured on the cells in the dark period as the potential activity.

cultures under the two conditions. The highest percentage of dividing cells was observed 3-4 h after the onset of illumination under aerobic conditions, but after $7-8 \mathrm{~h}$ under microaerobic/anaerobic conditions. During the light period of $12 \mathrm{~h}$ under aerobic conditions, photosynthetic activity was observed first, followed by the appearance of nitrogenase activity. In contrast, under microaerobic/anaerobic conditions, nitrogenase activity preceded the appearance of the peak photosynthetic activity. These observations were consistent throughout the three consecutive cell cycles (Figs 2 and 3).

In the microaerobic/anaerobic culture, the nitrogenase activity of cells at the transition from dark to light was significantly higher in the light assay than in the dark assay (Fig. 3). The nitrogenase activity was minimal during the dark period (Fig. 3). In the aerobic culture, the nitrogenase activity of cells assayed in the light and the dark at the transition did not differ substantially, although it tended to be slightly higher in the light assay (Fig. 2). In both conditions, nitrogenase activity was detected and increased to a certain level at the end of the $12 \mathrm{~h}$ light period. This was convenient for describing how the induced nitrogenase activity was affected by the imposition of darkness. By reducing the length of the light

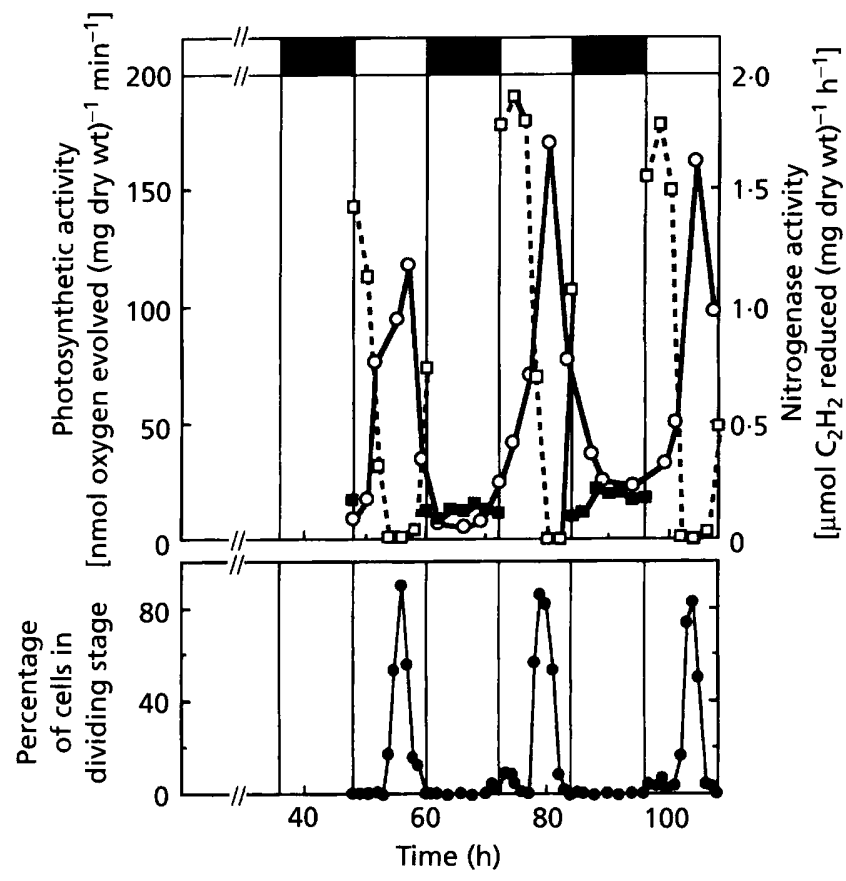

Fig. 3. Changes in nitrogen-fixing and photosynthetic activities of a culture placed in $12 \mathrm{~h}$ dark-12 h light cycles under anaerobic or microaerobic conditions. Inoculation was carried out at $0 \mathrm{~h}$. Aeration (nitrogen/carbon dioxide) was continued throughout the experiment. Nitrogenase activity was measured under an argon atmosphere as the rate of $\mathrm{C}_{2} \mathrm{H}_{2}$ reduction either in the light ( $\square$ ) or in the dark ( $\square$ ), or in both the light and the dark at the light/dark and dark/light transitions. Photosynthetic activity $(O)$ was measured as the rate of net oxygen evolution. The activity was also measured on the cells in the dark period as the potential activity.

period, we could place the cells in the dark before the nitrogenase activity increased (Figs 4 and 5). Thus, the change in carbohydrate content was simply evaluated in relation to nitrogenase activity.

Under a $14 \mathrm{~h}$ dark-10 h light regime, periodic cell division was observed under both aerobic (Fig. 4) and microaerobic/anaerobic (Fig. 5) conditions and occurred 3-4 h after the onset of the light period under aerobic conditions and $7-8 \mathrm{~h}$ under microaerobic/anaerobic conditions. At each peak, the percentage of dividing cells was more than $80 \%$ (data not shown). As with the $12 \mathrm{~h}$ light $-12 \mathrm{~h}$ dark regime, nitrogenase activity in microaerobic/anaerobic cultures was minimal during the dark period, but increased immediately upon the onset of illumination (Fig. 5). In the aerobic culture (Fig. 4), nitrogenase activity started to appear about $10 \mathrm{~h}$ after the beginning of the illuminated period, but it was not disturbed by the imposition of the dark period.

During the phase of active nitrogen fixation, substantial consumption of carbohydrate was observed (Figs 4 and 5). Under microaerobic/anaerobic conditions, nitrogenase activity had not appeared at the end of the light period. The activity was minimal during the dark period, though there was a gradual consumption of carbohydrate 


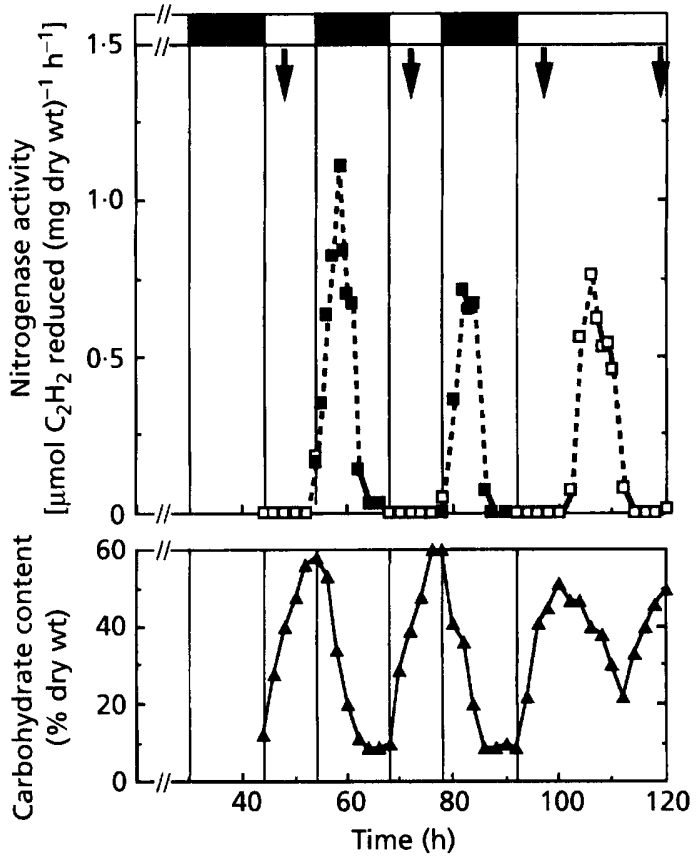

Fig. 4. Changes in nitrogenase activity and cellular carbohydrate content of a culture placed in $14 \mathrm{~h}$ dark-10 h light cycles under aerobic conditions. Inoculation was carried out at $0 \mathrm{~h}$. Aeration (air/carbon dioxide) was continued throughout the experiment. Nitrogenase activity was measured either in the light ( $\square$ ) or in the dark ( $\square$ ), or in both the light and the dark at the light/dark and dark/light transitions. Arrows indicate the time of synchronized cell division. The culture was placed under continuous illumination after the third dark period.

(Fig. 5). Carbohydrate accumulation occurred during the phase of active photosynthesis (data not shown). There was a considerable difference in the range of variation in cellular carbohydrate content between aerobic and microaerobic/anaerobic cultures. In the aerobic synchronous culture, it varied from $9 \%$ to $60 \%$ cell dry weight, but only from 12 to $42 \%$ cell dry weight in the microaerobic/anaerobic synchronous culture. The same levels of maximum and minimum carbohydrate content were repeatedly obtained in light-dark cycles. The time required for the increase of carbohydrate content to the maximum percentage was about $8-10 \mathrm{~h}$ in aerobic cultures, and $4-8 \mathrm{~h}$ in microaerobic/anaerobic cultures. Under aerobic dark conditions, it took $8-10 \mathrm{~h}$ for cellular carbohydrates to decline to their minimum level.

When the microaerobic/anaerobic synchronous culture was placed under continuous illumination, cell cycle events continued in series as if the dark period was omitted. The oscillatory changes in nitrogenase activity and cellular carbohydrate content continued, without the slow decline in carbohydrate characteristic of the dark phase (Fig. 5). The time required for the decrease of carbohydrate content to the minimum percentage was $6-8 \mathrm{~h}$ in this culture in the light (Fig. 5).

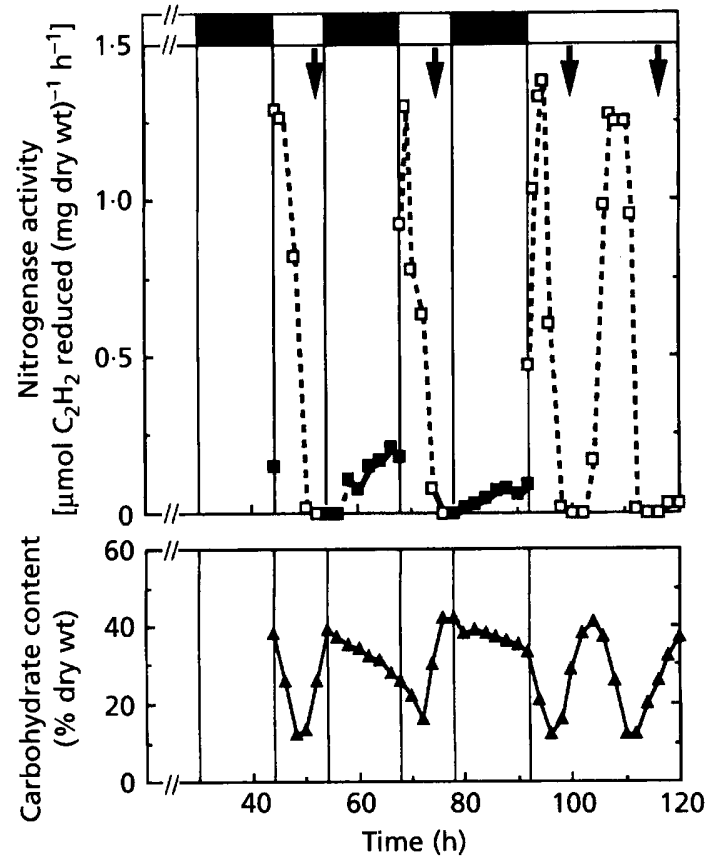

Fig. 5. Changes in nitrogenase activity and cellular carbohydrate content of a culture placed in $14 \mathrm{~h}$ dark-10 $\mathrm{h}$ light cycles under anaerobic or microaerobic conditions. Inoculation was carried out at $0 \mathrm{~h}$. Aeration (nitrogen/carbon dioxide) was continued throughout the experiment. Nitrogenase activity was measured either in the light $(\square)$ or in the dark ( $\square$ ), or in both the light and the dark at the light/dark and dark/light transitions. Arrows indicate the time of synchronized cell division. The culture was placed under continuous illumination after the third dark period.

\section{DISCUSSION}

Synecbococcus sp. Miami BG 043511 grew a little more slowly under aerobic than microaerobic/anaerobic conditions. However, the relatively small difference observed in this strain suggests that the effect of atmospheric oxygen does not seem to be detrimental to diazotrophic growth. A greater difference has been observed in Synechococcus sp. strain SF1, in which a generation time of $49 \mathrm{~h}$ under aerobic conditions was reduced to $38 \mathrm{~h}$ under anaerobic conditions (Spiller \& Shanmugam, 1987).

The application of light-dark cycles to microaerobically/ anaerobically growing, nitrogen-fixing cultures resulted in periodic peaks in cell division, nitrogen fixation and photosynthesis. Under aerobic conditions, the initial appearance of peak photosynthetic activity in the light period was followed by a peak of nitrogenase activity (Fig. 2). This result agrees with our previous observations in aerobic cultures when synchronous growth was induced prior to the application of the dark-light cycles (Mitsui et al., 1986). The sequence of the appearance of these activities was reversed in microaerobic/anaerobic cultures, with nitrogenase activity reaching a peak immediately after the onset of illumination (Fig. 3). Under both conditions, however, cell division was observed at or just after the peak of photosynthetic activity (Figs 2 and 
3). Thus, the timing of cell division under both conditions seemed to be consistent, relative to the peak of photosynthetic activity.

As reported in our previous studies for synchronously growing cultures of this strain under aerobic conditions (Mitsui et al., 1986, 1987; Suda et al., 1992), intensive degradation of carbohydrate was consistently observed during the phase of active nitrogenase activity in both cultures, whether this was in the light or dark. The degradation observed in the light is in contrast to the observations of Ortega-Calvo \& Stal (1991), who have shown that Gloeothece sp. grown diazotrophically in continuous culture exhibits little change in glycogen content. Little or minimum photosynthetic oxygen evolution was observed during the phase of active nitrogen fixation under both aerobic and microaerobic/anaerobic conditions. This coincides with the temporal separation of nitrogenase and photosynthetic activities reported previously (Mitsui et al., 1986; Kumazawa \& Mitsui, 1992). Thus, endogenous carbohydrate, rather than concurrent photosynthesis, seems to provide a source of electron donors for nitrogen fixation under both conditions.

The cyclic pattern of the change in cellular carbohydrate content showed distinct differences in the two cultures. Under dark anaerobic conditions (Fig. 5), there was a gradual degradation of carbohydrate, although it was much slower than that observed during the phase of active nitrogenase activity (Figs 4 and 5). Maryan et al. (1986) have shown that anaerobic dark conditions are not adequate to support nitrogenase activity in Gloeothece sp., due to the limitation of ATP supply, and nitrogenase activity under anaerobic conditions is therefore strictly light-dependent. In microaerobic/anaerobic synchronous cultures of strain Miami BG 043511, at the transition point from dark to light, there was a considerable difference between the nitrogenase activities assayed with or without light (Figs 3 and 5). Nitrogenase activity under dark anaerobic conditions was minimal, while cellular carbohydrate degradation slowly progressed even in the dark. In aerobic synchronous cultures of this strain under continuous illumination, addition of DCMU $(20 \mu \mathrm{M})$, which completely inhibits photosynthetic oxygen evolution, does not inhibit nitrogenase activity (Mitsui $e t a l$., 1986), hence the principal role of light in nitrogen fixation seems to be generation of ATP via photophosphorylation, rather than the supply of reductant.

The pattern of the appearance of nitrogenase activity was not disturbed when the culture entered the dark period (Figs 2 and 4). Since substantial nitrogenase activity was observed under aerobic dark conditions, respiratory generation of ATP seems to be sufficient to support nitrogen fixation in strain Miami BG 043511. Under aerobic dark conditions, therefore, endogenous carbohydrate plays important roles in generating ATP and reductant for nitrogenase catalysis. In Gloeothece sp., respiration plays a major role in the supply of ATP for nitrogen fixation under aerobic conditions even in the light (Maryan $e t$ al., 1986). When nitrogenase activity of strain Miami BG 043511 was assayed in the light and the dark under aerobic conditions, there was little difference at the transition from light to dark (Figs 2 and 4). This implies that nitrogenase activity of aerobically grown cultures does not require photophosphorylation. The absence of photosynthesis during the phase of active nitrogen fixation compels the cells to employ endogenous carbohydrates to supply reductants and ATP even in the light.

Anaerobic cultures of strain Miami BG 043511 do not fix nitrogen in the dark, presumably because of insufficient ATP generation. Synchronized anaerobic cultures must therefore fix nitrogen during the forthcoming light period by the aid of photophosphorylation. This creates a potential clash with photosynthetic oxygen evolution. However, the delay in the appearance of photosynthesis on return to the light permits a period of nitrogen fixation before oxygen concentrations become inhibitory. The difference in the sequence of photosynthesis and nitrogen fixation of the two synchronous cultures is consequently attributed to the ability to fix nitrogen in the dark. The phase of nitrogen fixation is carried over to the next light period under anaerobic conditions, while under aerobic conditions, cells complete nitrogen fixation in the dark, and are kept waiting for the resumption of photosynthesis.

The magnitude of the change in carbohydrate levels was higher in aerobic cultures than in microaerobic/anaerobic cultures (Figs 4 and 5). The minimum level of cellular carbohydrate was about $10 \%$ of cell dry weight under both conditions, but during the phase of photosynthesis, carbohydrate content increased to $60 \%$ of cell dry weight in aerobic cultures, and only $40 \%$ in microaerobic/ anaerobic cultures. These percentages were repeatedly observed during dark-light cycles of well-synchronized cultures in which the oscillatory changes in nitrogenase and photosynthetic activities showed sharp peaks and troughs. These results imply that cells in aerobic cultures need larger amounts of reserve polysaccharides to satisfy the demand of nitrogen fixation than those in microaerobic/anaerobic cultures.

When a synchronized population of strain Miami BG 043511 is placed in continuous light under aerobic conditions, alternating peaks in nitrogenase and photosynthetic activity continue for three cell cycles, although the synchrony is gradually lost (Mitsui et al., 1987). When synchronous cultures growing under microaerobic/ anaerobic conditions are placed in continuous light, the alternating phases of nitrogen fixation and photosynthesis continue for at least two cycles (Fig. 5). However, in asynchronous aerobic or microaerobic batch cultures, these cycles are not apparent in the culture as a whole, but presumably continue in individual cells. Since the level of maximum carbohydrate content was higher, the periods of both carbohydrate accumulation and consumption were longer in aerobic cultures $(8-10 \mathrm{~h})$ than microaerobic/anaerobic cultures $(4-8 \mathrm{~h})$. This might result in a longer cell cycle for cells in asynchronous aerobic cultures. It may be this difference in cell cycle length, rather than oxygen inhibition of nitrogenase, 
which explains the slower growth rate of aerobic cultures (Fig. 1).

\section{ACKNOWLEDGEMENTS}

We are grateful to Dr S. Kumazawa (Tokai University) and Ms C. Campbell (University of Miami) for comments and suggestions. This work was supported in part by grants given to A. Mitsui from the US National Science Foundation, the US Department of Energy - NREL, Clean Energy Institute of Japan and NEDO-RITE of Japan.

This study constitutes part of dissertation work by $\mathrm{H}$. Ikemoto which was performed at the University of Miami.

\section{REFERENCES}

Dubois, M., Gilles, K. A., Hamilton, K., Rebers, P. A. \& Smith, F. (1956). Colorimetric method for determination of sugars and related substances. Anal Chem 28, 350-356.

Fay, P. (1992). Oxygen relations of nitrogen fixation in cyanobacteria. Microbiol Rev 56, 340-373.

Gallon, J. R. (1992). Reconciling the incompatible: $\mathrm{N}_{2}$ fixation and $\mathrm{O}_{2}$. New Phytol 122, 571-609.

Kumazawa, S. \& Mitsui, A. (1985). Comparative study of uptake hydrogenase and hydrogen photoproduction activities between heterocystous cyanobacterium Anabaena cylindrica B629 and nonheterocystous cyanobacterium Oscillatoria sp. strain Miami BG 7. Appl Environ Microbiol 50, 287-291.

Kumazawa, S. \& Mitsui, A. (1992). Photosynthetic activities of a synchronously grown aerobic $\mathrm{N}_{2}$-fixing unicellular cyanobacterium, Synechococcus sp. Miami BG 043511. J Gen Microbiol 138, $467-472$

Leon, C., Kumazawa, S. \& Mitsui, A. (1986). Cyclic appearance of aerobic nitrogenase activity during synchronous growth of unicellular cyanobacteria. Curr Microbiol 13, 149-153.
Maryan, P. S., Eady, R. R., Chaplin, A. E. \& Gallon, J. R. (1986). Nitrogen fixation by Gloeothece sp. PCC 6909: respiration and not photosynthesis supports nitrogenase activity in the light. $J$ Gen Microbiol 132, 789-796.

Mitsui, A. \& Cao, S. (1988). Isolation and culture of marine nitrogen-fixing unicellular cyanobacteria Synechococcus. Methods Enzymol 167, 105-113.

Mitsui, A., Phlips, E. J., Kumazawa, S., Reddy, K. J., Ramachandran, S., Matsunaga, T., Haynes, L. \& Ikemoto, H. (1983). Progress in research toward outdoor biological hydrogen production using solar energy, sea water, and marine photosynthetic microorganisms. Ann NY Acad Sci 413, 514-530.

Mitsui, A., Kumazawa, S., Takahashi, A., Ikemoto, H., Cao, S. \& Arai, T. (1986). Strategy by which nitrogen-fixing unicellular cyanobacteria grow photoautotrophically. Nature 323, 720-722.

Mitsui, A., Cao, S., Takahashi, A. \& Arai, T. (1987). Growth synchrony and cellular parameters of the unicellular nitrogen-fixing marine cyanobacterium, Synechococcus sp. strain Miami BG 043511 under continuous illumination. Pbysiol Plant 69, 1-8.

Mitsui, A., Suda, S. \& Hanagata, N. (1993). Cell cycle events at different temperatures in aerobic nitrogen-fixing marine unicellular cyanobacterium Synechococcus sp. Miami BG 043511. J Mar Biotechnol 1, 89-91.

Ortega-Calvo, J.-J. \& Stal, L. J. (1991). Diazotrophic growth of the unicellular cyanobacterium Gloeothece sp. PCC 6909 in continuous culture. J Gen Microbiol 137, 1789-1797.

Spiller, H. \& Shanmugam, K. T. (1987). Physiological conditions for nitrogen fixation in a unicellular marine cyanobacterium, Synechococcus sp. strain SF1. J Bacteriol 169, 5379-5384.

Suda, S., Kumazawa, S. \& Mitsui, A. (1992). Change in the $\mathrm{H}_{2}$ photoproducing capability in a synchronously grown aerobic nitrogen-fixing cyanobacterium, Synechococcus sp. Miami BG 043511. Arch Microbiol 158, 1-4.

Received 1 October 1993; revised 14 March 1994; accepted 31 March 1994. 